\title{
Probing the Epoch of Reionisation with the MWA
}

\section{Christopher H. Jordan*}

International Centre for Radio Astronomy Research (ICRAR), Curtin University, Bentley WA, Australia

ARC Centre of Excellence for All Sky Astrophysics in 3 Dimensions (ASTRO 3D), Australia

E-mail: christopher.jordan@curtin.edu.au

\section{Cathryn M. Trott}

International Centre for Radio Astronomy Research (ICRAR), Curtin University, Bentley WA, Australia

ARC Centre of Excellence for All Sky Astrophysics in 3 Dimensions (ASTRO 3D), Australia

E-mail: cathryn.trottecurtin.edu.au

\section{Christene Lynch}

International Centre for Radio Astronomy Research (ICRAR), Curtin University, Bentley WA, Australia

ARC Centre of Excellence for All Sky Astrophysics in 3 Dimensions (ASTRO 3D), Australia E-mail: christene. Iynchecurtin.edu.au

\section{Jack L. B. Line}

International Centre for Radio Astronomy Research (ICRAR), Curtin University, Bentley WA, Australia

ARC Centre of Excellence for All Sky Astrophysics in 3 Dimensions (ASTRO 3D), Australia

E-mail: jack.line@curtin.edu.au

The Epoch of Reionisation (EoR) is a crucial piece of cosmic history that has thus far eluded detection. In these proceedings, we detail our approach to its detection with the Murchison Widefield Array radio telescope in the remote Western Australian desert.

The 4th KMI International Symposium (KMI2019)

18-20, February 2019

Nagoya, Japan

${ }^{*}$ Speaker. 


\section{Introduction}

The Epoch of Reionisation (EoR) is a period of cosmological time in which the first stars and galaxies were formed and began to ionise the surrounding neutral intergalactic medium approximately 400 million years after the Big Bang. One important aspect of the EoR is its ability to connect what happened shortly after the Big Bang to a completely ionised universe; this would allow us to attribute the variations in the Cosmic Microwave Background (CMB) with the evolution of matter, forming the first stars and galaxies, how they affect each other over time, and finally the evolution of all galaxies in the observable universe.

However, gleaning details from the EoR has thus far proven difficult. As the "signature" of the EoR is quite old, and therefore weak, measuring the signature of the EoR requires a deep understanding of all other signals earlier than that of the EoR. In this field of research, we term these other signals as foregrounds.

One of the most popular targets for studying the EoR is the hyperfine $21 \mathrm{~cm}$ spectral line of neutral hydrogen. This spectral line is weak, as a single atom on average emits every 11 million years. Neutral hydrogen is a relatively abundant substance, even (as is expected) during the EoR, which helps to counter-balance the poor strength of the spectral line. However, as this line is targeted at high redshifts (typically $z \sim 6$ ), the line strength is further diminished, and its wavelength is elongated into the low-frequency astronomy regime $(<200 \mathrm{MHz})$. It is expected that, at these frequencies, the foregrounds to the EoR are approximately $10^{5}$ times brighter than that of the EoR signature. To reach the required sensitivity for EoR detection, a large amount of data is required (at least 1,000 hours [2]). In addition, low-frequency astronomy poses its own suite of challenges on top of those facing "conventional" radio astronomy, including significant ionospheric contamination, which are detailed further in these proceedings.

With the Murchison Widefield Array [13] (MWA; a low-frequency radio telescope), we aim to statistically detect the signature of Epoch of Reionisation by collecting thousands of hours of data, understanding and precisely calibrating the MWA, and modelling and removing any foreground components. The statistical detection of the spatial structure of the $21 \mathrm{~cm}$ brightness temperature fluctuations is the primary aim of most current instruments, owing to its natural relationship to the measurement space of an interferometer (Fourier modes), the increase in sensitivity it affords, and the assumption that the signal is isotropic.

\section{The Murchison Widefield Array}

The MWA Phase I is a 128-element dipole array located in the Murchison shire of Western Australia, which is isolated from human-made radio interference, and is capable of observing frequencies between 80 and $300 \mathrm{MHz}$. At an observing frequency of $200 \mathrm{MHz}$, the primary beam is approximately $20 \times 20$ square degrees in size, which is an unusually large field of view in radio astronomy. The MWA has four main science projects [3], one of which is EoR science, and was designed with EoR detection in mind. Furthermore, the MWA is also a Square Kilometre Array (SKA) precursor, which acts as the next generation of radio astronomy instrumentation seeking to answer the biggest questions in the field, including those involved with the EoR [8]. 
Phase I of the MWA [13,3] operated from 2013 to 2016, and comprised 128 tiles spread over $3 \mathrm{~km}$. The core of the array contained a large filling fraction, to provide excellent snapshot imaging capability and sensitivity to EoR angular scales. Phase II of the MWA [18] has operated from 2016 and expanded the array to 256 tiles spread over $5 \mathrm{~km}$. Of most importance for EoR science was the addition of two redundant sub-arrays in hexagonal configurations allowing for improved calibration and extra sensitivity to relevant EoR scales. Figure 2 (left) shows the array layout for Phase II (red), with a cutout for the 72 subarrays (blue), and (right) the sensitivity for Phase I (red) and II (green) for a $1000 \mathrm{~h}$ experiment at $z=8.5$.
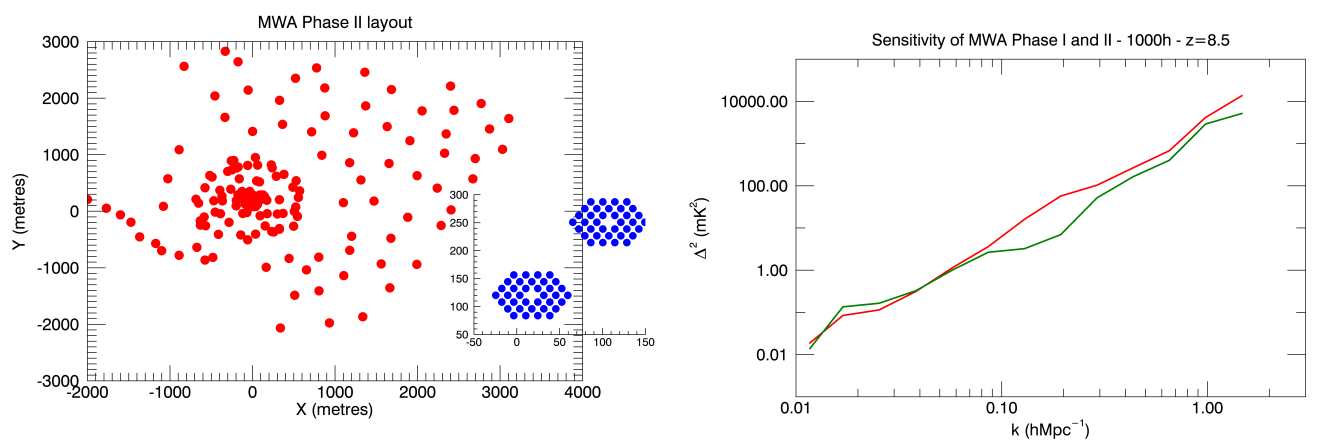

Figure 1: (left) shows the array layout for Phase II (red), with a cutout for the 72 subarrays (blue), and (right) the sensitivity for Phase I (red) and II (green) for a $1000 \mathrm{~h}$ experiment at $z=8.5$.

\subsection{Calibration of MWA Data}

Data from the MWA are typically stored as two-minute "snapshots", containing two-second integrations at a frequency resolution of $40 \mathrm{kHz}$ over $30.72 \mathrm{MHz}$ of bandwidth. The majority of EoR observations are taken in either the "high-" or "low-band" (183 and $155 \mathrm{MHz}$, respectively) and are pointed at either the "EoR- -0 " $\left(\alpha=0^{\mathrm{h}}, \delta=-27^{\circ}\right)$ or "EoR-1" field $\left(\alpha=4^{\mathrm{h}}, \delta=-27^{\circ}\right)$. There exist a few different methods to calibrate the data for science purposes, however, in these proceedings we focus on the Real-Time System (RTS; [11]) software, which is one of two software packages extensively used for MWA EoR data.

The primary aspect of the RTS is to run MWA data through a "calibrate-measure-loop" (CML). A brief description of the CML is as follows:

(i) A catalogue of visible radio sources and their flux densities are ranked according to the antenna primary beam pattern of the observation. The highest-ranked sources are referred to as calibrators for the remainder of the CML;

(ii) To mitigate sidelobe contamination in visibility space, the calibrators are subtracted ("prepeeled");

(iii) Looping over every calibrator, the phase centre of the visibility space is rotated such that the pointing centre is the location of the current calibrator, and its visibilities are re-added;

(iv) To determine any refraction and scintillation of the calibrator due to the ionosphere, a wavelength-squared fit is applied, and the phase centre is adjusted;

(v) Assuming the quality of the data is adequate, the calibrator is "peeled" (subtracted and not restored) from the observation. Continue looping from step (iii). 
The RTS method of peeling sources from an observation is how the MWA EoR team is able to remove foreground sources, in the hopes of reaching the weak, elusive EoR signature. As mentioned, peeling requires fitting of any ionospheric refraction or scintillation, which is extremely useful for studying the ionosphere, which in turn is useful for quality control of EoR data, such that we can avoid observations where the ionosphere is relatively active. However, peeling is not possible without accurate source models over a large portion of the sky (especially those in and surrounding the aforementioned EoR fields). For this reason, a lot of work has gone into improving source models and better understanding the interaction between the instrument, data and software.

\section{Recent developments with MWA EoR}

\subsection{The Long Baseline Epoch of Reionisation Survey}

The Long Baseline Epoch of Reionisation Survey (LoBES) aims to improve our source modelling by using the extended phase II configuration of the MWA to target the EoR- 0 and -1 fields, as well their surrounding sky fields. With the additional resolution afforded by the phase II configuration, an upcoming publication (Lynch et al. in prep.) shows that double sources which have been incorrectly modelled as single sources can approximately double the amount of residual power leftover after calibration. This work will improve the peeling performed by the RTS, and ultimately bring our experiment closer to an EoR detection.
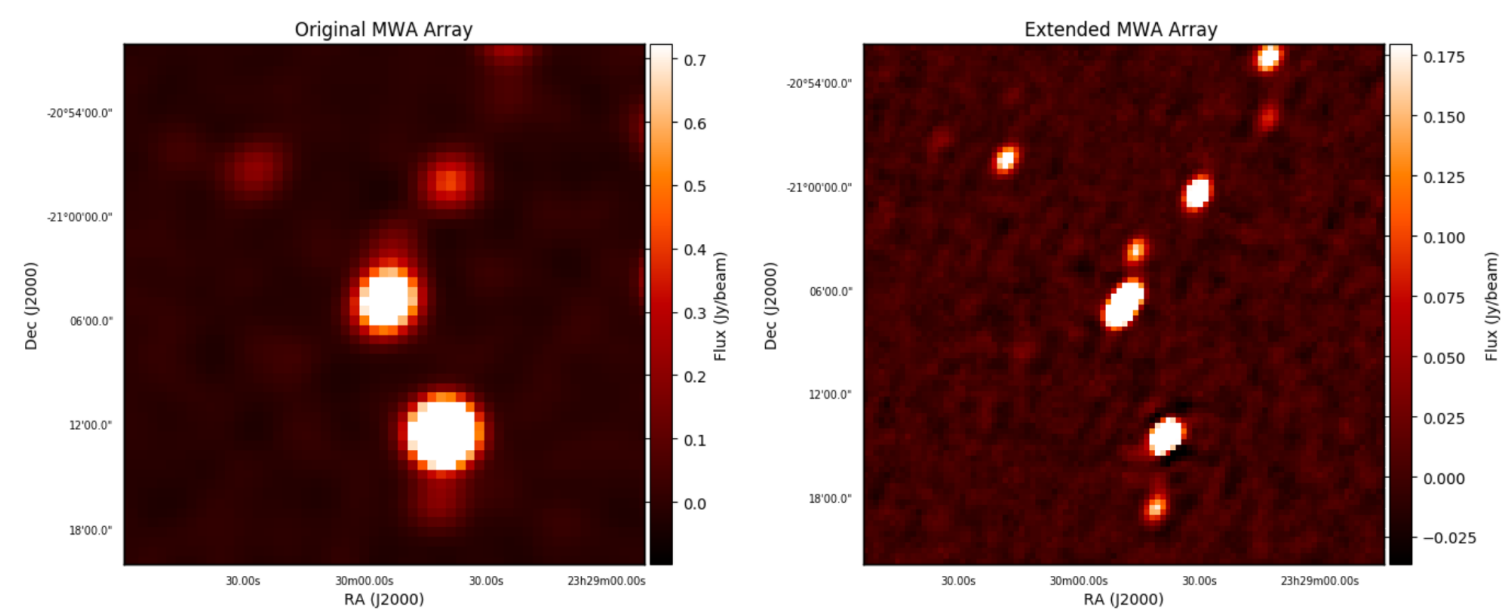

Figure 2: MWA images of a patch of sky from both phase I ("Original MWA Array") and phase II extended ("Extended MWA Array") configurations. The extended array is able to identify double sources that the original array could not.

\subsection{Source modelling with Fornax A}

Fornax A is a bright galaxy within the EoR-1 field, and is a good example of a complicated foreground source that must be subtracted from EoR data. For MWA calibration, most sources are modelled as simple Gaussian point sources. It is possible to model Fornax A using many Gaussians; however, using shapelets, an upcoming publication (Line et al. in prep.) shows that we are able to accurately model Fornax A and substantially reduce our computation time required to remove 
it from EoR data. At present, a typical calibration of an EoR observation requires 20 minutes of supercomputing time for each two-minute snapshot; when averaging hundreds or thousands of hours worth of data, this development will significantly help our efforts toward an EoR detection.
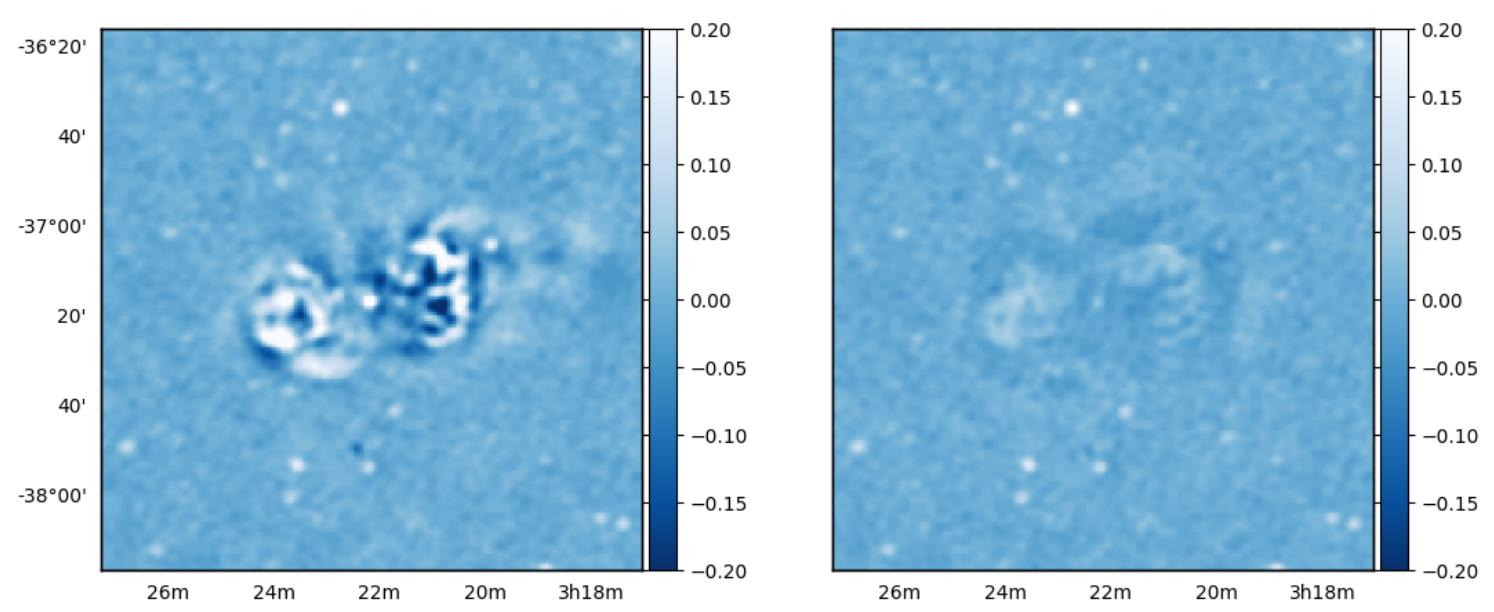

Figure 3: MWA images of the Fornax A galaxy after being peeled with an old shapelets model (left) and after being peeled with a new shapelets model (right). There is a significant improvement between the models, and while small artefacts remain in the image on the right, these comprise a very small fraction of the total flux density of the foreground source.

\subsection{EoR power limits}

There are two primary data calibration and analysis pipelines that MWA EoR data are processed through, and results compared. The RTS, based in Australia, outputs calibrated and sourcesubtracted visibilities (Fourier modes) as a function of frequency, and these are fed through CHIPS [14], a power spectrum estimator that uses knowledge of the instrument response function to estimate the power in different spatial modes, and their associated uncertainties. Based in North America, Fast Holographic Deconvolution (FHD; [12]) approaches data calibration and source subtraction using a different technique to RTS, but with equivalent outputs. Output images are fed through $\varepsilon$ ppsilon, as the power spectrum estimator [1]. A novel and important feature of the MWA data analysis is to feed the same data independently through both pipelines, in order to ensure robustness of results. The philosophical structure of this approach, and its details, are described in [6].

These two primary pipelines have been used to publish the most recent deep EoR limits from the MWA Phase I. In [2], both pipelines processed a matched set of 3-hours of data from the 2013 observing season of the EoR-0 field and the high-band $(z=6.5-8)$, reaching consistent limits. More recently, both pipelines have been used with improvements to process Phase II to deeper limits. These papers are currently under review.

\subsection{Ionospheric analyses}

The ionosphere is the outer-most layer of the Earth's atmosphere, and affects low-frequency radio astronomy by altering the properties of the sky, primarily through refraction and scintillation. 
This is problematic for radio astronomy in general, but especially so for the EoR experiment, where care must be taken to coherently average data without ionospheric contamination.

As discussed earlier, the RTS is able to measure the contribution of the ionosphere toward each calibrator source when calibrating an observation. Coupled with the relatively large field-ofview offered by the MWA, the RTS is an excellent tool for studying the effects of the ionosphere. A preliminary investigation [7] found that approximately 74 percent of observations have relatively little ionospheric contamination, and divided the remaining observations into another three types of ionospheric activity, suggesting the severity of their impact on MWA data. Another publication [16] confirms that ionospherically-active data, especially those with coherent structures, should be avoided for the purposes of an EoR detection.

In order to avoid poor-quality observations, we have created a quality assurance database to keep track of various metrics, including ionospheric activity. With its population of many thousands of observations, an upcoming publication (Jordan et al. in prep.) will further detail the statistics of ionospheric activity and its behaviour over time.

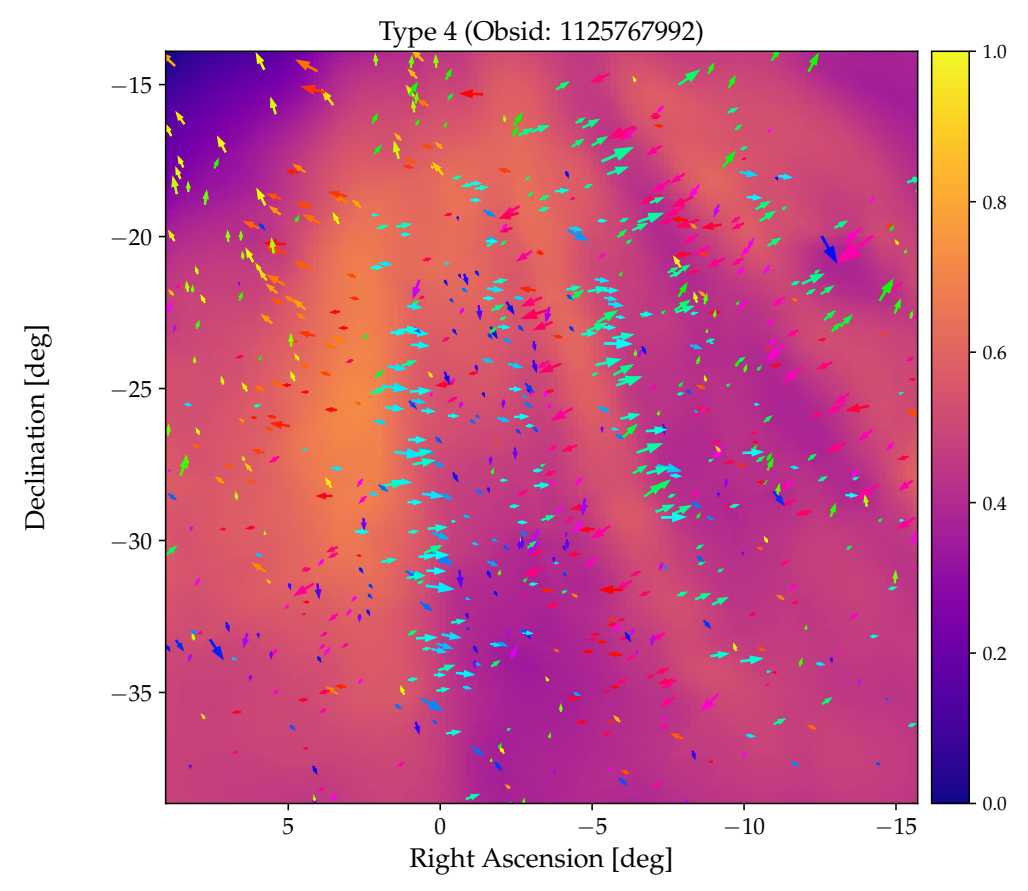

Figure 4: An example of extreme ionospheric activity (Type 4) found in [7]. The vectors designate the ionospheric refractions measured by the RTS. Using a spatial reconstruction method, the background image is produced by the vectors, which aids identification of ionospheric structure.

\subsection{Statistics beyond the power spectrum}

Although the spatial power spectrum is the primary data product of most current EoR $21 \mathrm{~cm}$ experiments, there are other avenues of pursuit to explore this first billion years of the Universe. Direct imaging is beyond the capability of current instruments, demanding a high surface bright- 
ness sensitivity and thousands of hours. This will be pursued by the future SKA [8]. However, there are other statistics that can be pursued through the $21 \mathrm{~cm}$ line, and also the opportunity for cross-correlating the signal with other tracers of early Universe evolution. The benefit of the latter approach is that the systematic errors may be different between the two tracers, offering an advantage over $21 \mathrm{~cm}$ alone.

The brightness temperature of the $21 \mathrm{~cm}$ line, relative to the $\mathrm{CMB}$, is determined by a range of physical factors, including the local properties of the gas temperature and the radiation field $[5,10$, 4]. At early times, it traces the matter power spectrum, and is highly Gaussian, but at later times the evolution of ionised bubbles dominates the spatial fluctuations and the signal is expected to have non-zero higher order terms. The shape of the temperature distribution function evolves with time and spatial scale, and differs for different underlying models of the evolution of the Universe. As such, probing these non-Gaussian components can provide complementary information to the power spectrum, which, by design, only captures information in the second moment of the distribution [19]. The bispectrum is one such statistic, and it has been shown to encode non-Gaussianity by measuring the three-point correlation function.

In early work to study the expected sensitivity of $21 \mathrm{~cm}$ experiments to the bispectrum, [22] computed expectations for a range of instruments, under the assumption of thermal noise only. More sophisticated recent work included the effects of calibration and foregrounds on the ability to detect the signal. In [17], two bispectrum estimators were developed that transitioned the study from theoretical to practical, and were applied to 20 hours of data from the Phase II of the MWA. This work discussed some of the advantages and challenges of doing such an experiment with real data. Bispectrum estimates with data from the Cosmic Dawn observed with the MWA and currently being undertaken (Yoshiura, priv. comm.).

Cross-correlation studies from the early Universe offer the potential for new astrophysical insight and reduced observational biases and errors. In the context of the MWA, [20] used data to explore the cross-correlation of the $21 \mathrm{~cm}$ image from the EoR- 0 observing field, and the CMB field measured by Planck. An additional tracer that can be used is the population of high-redshift Ly- $\alpha$ emitters (LAEs), which are observable in ionised regions [21, 9].

Beyond new analyses the extract cosmological information, the large systematic impediments presented currently by foreground signals demands careful attention. In [15], the EoR group used a kernel density estimator to study the distribution of data in different wavemodes from two different observing fields. This work showed that foregrounds differed between locations on the sky, potentially offering an avenue for future use of such estimators to discriminate isotropic cosmological signal from local foreground contamination.

\section{Future outlook}

The MWA EoR project continues to perfect its understanding of MWA instrumentation as well as its calibration, and is seeing improvements in the one-dimensional power spectrum. In time, we expect to have made enough progress to overcome the challenging EoR detection. 


\section{References}

[1] N. Barry, A. P. Beardsley, R. Byrne, B. Hazelton, M. F. Morales, J. C. Pober, and I. Sullivan. The FHD/cppsilon Epoch of Reionization Power Spectrum Pipeline. arXiv e-prints, page arXiv:1901.02980, Jan 2019.

[2] A. P. Beardsley, B. J. Hazelton, I. S. Sullivan, P. Carroll, N. Barry, M. Rahimi, B. Pindor, C. M. Trott, J. Line, D. C. Jacobs, M. F. Morales, J. C. Pober, G. Bernardi, J. D. Bowman, M. P. Busch, F. Briggs, R. J. Cappallo, B. E. Corey, A. de Oliveira-Costa, J. S. Dillon, D. Emrich, A. Ewall-Wice, L. Feng, B. M. Gaensler, R. Goeke, L. J. Greenhill, J. N. Hewitt, N. Hurley-Walker, M. Johnston-Hollitt, D. L. Kaplan, J. C. Kasper, H. S. Kim, E. Kratzenberg, E. Lenc, A. Loeb, C. J. Lonsdale, M. J. Lynch, B. McKinley, S. R. McWhirter, D. A. Mitchell, E. Morgan, A. R. Neben, N. Thyagarajan, D. Oberoi, A. R. Offringa, S. M. Ord, S. Paul, T. Prabu, P. Procopio, J. Riding, A. E. E. Rogers, A. Roshi, N. Udaya Shankar, S. K. Sethi, K. S. Srivani, R. Subrahmanyan, M. Tegmark, S. J. Tingay, M. Waterson, R. B. Wayth, R. L. Webster, A. R. Whitney, A. Williams, C. L. Williams, C. Wu, and J. S. B. Wyithe. First Season MWA EoR Power Spectrum Results at Redshift 7. ArXiv e-prints, August 2016.

[3] J. D. Bowman, I. Cairns, D. L. Kaplan, T. Murphy, D. Oberoi, and others. Science with the Murchison Widefield Array. PASA, 30:31, April 2013.

[4] D. J. Eisenstein and W. Hu. Power Spectra for Cold Dark Matter and Its Variants. ApJ, 511:5-15, January 1999.

[5] S. R. Furlanetto, S. P. Oh, and F. H. Briggs. Cosmology at low frequencies: The $21 \mathrm{~cm}$ transition and the high-redshift Universe. Phys. Rep., 433:181-301, October 2006.

[6] D. C. Jacobs, B. J. Hazelton, C. M. Trott, J. S. Dillon, B. Pindor, I. S. Sullivan, J. C. Pober, N. Barry, A. P. Beardsley, G. Bernardi, J. D. Bowman, F. Briggs, R. J. Cappallo, P. Carroll, B. E. Corey, A. de Oliveira-Costa, D. Emrich, A. Ewall-Wice, L. Feng, B. M. Gaensler, R. Goeke, L. J. Greenhill, J. N. Hewitt, N. Hurley-Walker, M. Johnston-Hollitt, D. L. Kaplan, J. C. Kasper, H. Kim, E. Kratzenberg, E. Lenc, J. Line, A. Loeb, C. J. Lonsdale, M. J. Lynch, B. McKinley, S. R. McWhirter, D. A. Mitchell, M. F. Morales, E. Morgan, A. R. Neben, N. Thyagarajan, D. Oberoi, A. R. Offringa, S. M. Ord, S. Paul, T. Prabu, P. Procopio, J. Riding, A. E. E. Rogers, A. Roshi, N. Udaya Shankar, S. K. Sethi, K. S. Srivani, R. Subrahmanyan, M. Tegmark, S. J. Tingay, M. Waterson, R. B. Wayth, R. L. Webster, A. R. Whitney, A. Williams, C. L. Williams, C. Wu, and J. S. B. Wyithe. The Murchison Widefield Array $21 \mathrm{~cm}$ Power Spectrum Analysis Methodology. ApJ, 825:114, July 2016.

[7] C. H. Jordan, S. Murray, C. M. Trott, R. B. Wayth, D. A. Mitchell, M. Rahimi, B. Pindor, P. Procopio, and J. Morgan. Characterization of the ionosphere above the Murchison Radio Observatory using the Murchison Widefield Array. MNRAS, 471:3974-3987, November 2017.

[8] L. Koopmans, J. Pritchard, G. Mellema, J. Aguirre, K. Ahn, R. Barkana, I. van Bemmel, G. Bernardi, A. Bonaldi, F. Briggs, and others. The Cosmic Dawn and Epoch of Reionisation with SKA. Advancing Astrophysics with the Square Kilometre Array (AASKA14), page 1, 2015.

[9] Kenji Kubota, Shintaro Yoshiura, Keitaro Takahashi, Kenji Hasegawa, Hidenobu Yajima, Masami Ouchi, B. Pindor, and R. L. Webster. Detectability of the 21-cm signal during the epoch of reionization with 21-cm Lyman $\alpha$ emitter cross-correlation - I. MNRAS, 479(2):2754-2766, Sep 2018.

[10] M. McQuinn, O. Zahn, M. Zaldarriaga, L. Hernquist, and S. R. Furlanetto. Cosmological Parameter Estimation Using $21 \mathrm{~cm}$ Radiation from the Epoch of Reionization. ApJ, 653:815-834, December 2006. 
[11] D. A. Mitchell, L. J. Greenhill, R. B. Wayth, R. J. Sault, C. J. Lonsdale, R. J. Cappallo, M. F. Morales, and S. M. Ord. Real-Time Calibration of the Murchison Widefield Array. IEEE Journal of Selected Topics in Signal Processing, 2:707-717, November 2008.

[12] I. S. Sullivan, M. F. Morales, B. J. Hazelton, W. Arcus, D. Barnes, G. Bernardi, F. H. Briggs, J. D. Bowman, J. D. Bunton, and R. J. Cappallo. Fast Holographic Deconvolution: A New Technique for Precision Radio Interferometry. ApJ, 759(1):17, Nov 2012.

[13] S. J. Tingay, R. Goeke, J. D. Bowman, D. Emrich, and others. The Murchison Widefield Array: The Square Kilometre Array Precursor at Low Radio Frequencies. PASA, 30:7, January 2013.

[14] C. M. Trott, B. Pindor, P. Procopio, R. B. Wayth, D. A. Mitchell, B. McKinley, S. J. Tingay, N. Barry, A. P. Beardsley, G. Bernardi, J. D. Bowman, F. Briggs, R. J. Cappallo, P. Carroll, A. de Oliveira-Costa, J. S. Dillon, A. Ewall-Wice, L. Feng, L. J. Greenhill, B. J. Hazelton, J. N. Hewitt, N. Hurley-Walker, M. Johnston-Hollitt, D. C. Jacobs, D. L. Kaplan, H. S. Kim, E. Lenc, J. Line, A. Loeb, C. J. Lonsdale, M. F. Morales, E. Morgan, A. R. Neben, N. Thyagarajan, D. Oberoi, A. R. Offringa, S. M. Ord, S. Paul, J. C. Pober, T. Prabu, J. Riding, N. Udaya Shankar, S. K. Sethi, K. S. Srivani, R. Subrahmanyan, I. S. Sullivan, M. Tegmark, R. L. Webster, A. Williams, C. L. Williams, C. Wu, and J. S. B. Wyithe. CHIPS: The Cosmological H i Power Spectrum Estimator. ApJ, 818:139, February 2016.

[15] Cathryn M. Trott, Shih Ching Fu, S. G. Murray, C. H. Jordan, J. L. B. Line, N. Barry, R. Byrne, B. J. Hazelton, K. Hasegawa, and R. Joseph. Robust statistics towards detection of the $21 \mathrm{~cm}$ signal from the Epoch of Reionization. MNRAS, 486(4):5766-5784, Jul 2019.

[16] Cathryn M. Trott, C. H. Jordan, S. G. Murray, B. Pindor, D. A. Mitchell, R. B. Wayth, J. Line, B. McKinley, A. Beardsley, and J. Bowman. Assessment of Ionospheric Activity Tolerances for Epoch of Reionization Science with the Murchison Widefield Array. ApJ, 867(1):15, Nov 2018.

[17] Cathryn M. Trott, Catherine A. Watkinson, Christopher H. Jordan, Shintaro Yoshiura, Suman Majumdar, N. Barry, R. Byrne, B. J. Hazelton, K. Hasegawa, and R. Joseph. Gridded and direct Epoch of Reionisation bispectrum estimates using the Murchison Widefield Array. arXiv e-prints, page arXiv:1905.07161, May 2019.

[18] R. B. Wayth, S. J. Tingay, C. M. Trott, D. Emrich, M. Johnston-Hollitt, B. McKinley, B. M. Gaensler, A. P. Beardsley, T. Booler, B. Crosse, T. M. O. Franzen, L. Horsley, D. L. Kaplan, D. Kenney, M. F. Morales, D. Pallot, G. Sleap, K. Steele, M. Walker, A. Williams, C. Wu, I. H. Cairns, M. D. Filipovic, S. Johnston, T. Murphy, P. Quinn, L. Staveley-Smith, R. Webster, and J. S. B. Wyithe. The Phase II Murchison Widefield Array: Design Overview. ArXiv e-prints, September 2018.

[19] J. S. B. Wyithe and M. F. Morales. Biased reionization and non-Gaussianity in redshifted 21-cm intensity maps of the reionization epoch. MNRAS, 379:1647-1657, August 2007.

[20] S. Yoshiura, K. Ichiki, B. Pindor, K. Takahashi, H. Tashiro, and C. M. Trott. Study of systematics effects on the cross power spectrum of $21 \mathrm{~cm}$ line and cosmic microwave background using Murchison Widefield Array data. MNRAS, 483(2):2697-2711, Feb 2019.

[21] S. Yoshiura, J. L. B. Line, K. Kubota, K. Hasegawa, and K. Takahashi. Detectability of $21 \mathrm{~cm}-$ signal during the Epoch of Reionization with $21 \mathrm{~cm}-L y m a n-\alpha$ emitter cross-correlation - II. Foreground contamination. MNRAS, 479(2):2767-2776, Sep 2018.

[22] Shintaro Yoshiura, Hayato Shimabukuro, Keitaro Takahashi, Rieko Momose, Hiroyuki Nakanishi, and Hiroshi Imai. Sensitivity for $21 \mathrm{~cm}$ bispectrum from Epoch of Reionization. MNRAS, 451(1):266-274, Jul 2015. 